\title{
The Modeling Framework for Experimental Physics: Description, development, and applications
}

\author{
Dimitri R. Dounas-Frazer and H. J. Lewandowski \\ Department of Physics, University of Colorado Boulder, Boulder, CO, USA \\ JILA, National Institute of Standards and Technology and University of Colorado \\ Boulder, Boulder, CO, USA \\ E-mail: dimitri.dounasfrazer@colorado.edu \\ E-mail: lewandoh@colorado.edu
}

29 May 2018

\begin{abstract}
The ability to construct, use, and revise models is a crucial experimental physics skill. Many existing frameworks describe modeling in science education at introductory levels. However, most have limited applicability to the context of upperdivision physics lab courses or experimental physics. Here, we discuss the Modeling Framework for Experimental Physics, a theoretical framework tailored to labs and experimentation. A key feature of the Framework is recursive interaction between models and apparatus. Models are revised to account for new evidence produced by apparatus, and apparatus are revised to better align with the simplifying assumptions of models. Another key feature is the distinction between the physical phenomenon being investigated and the measurement equipment used to conduct the investigation. Models of physical systems facilitate explanation or prediction of phenomena, whereas models of measurement systems facilitate interpretation of data. We describe the Framework, provide a chronological history of its development, and summarize its applications to research and curricular design. Ultimately, we argue that the Modeling Framework is a theoretically sound and well-tested tool that is applicable to multiple physics domains and research purposes. In particular, it is useful for characterizing students' approaches to experimentation, designing or evaluating curricula for lab courses, and developing instruments to assess students' experimental modeling skills.
\end{abstract}

Submitted to: Eur. J. Phys.

\section{Models and modeling in physics education}

The importance of modeling in physics education has been recognized for over 30 years. Johnson-Laird (1980), Hestenes (1987), and Halloun \& Hestenes (1987) played key roles in the development of early theories of learning and curricular design. Today, students' development of mental models (Brewe et al 2018, Körhasan et al 2016, Özcan 2015) and representational competence (Fredlund et al 2012, McPadden 
\& Brewe 2017, Opfermann et al 2017) continue to be active areas of research in lecture or studio settings. Meanwhile, undergraduate physics lab courses have been gaining increased attention over the last decade (Schumacher 2007). Consider, for example, multiple large survey studies of students' views about lab courses (Coppens et al 2016, Deacon \& Hajek 2011, Hanif et al 2009) or experimental physics (Wilcox \& Lewandowski 2018). Despite these trends, research on student engagement in modeling during lab courses is sparse, and there is a need for theoretical frameworks and empirical studies that focus on students' ability to model experimental systems.

Since the 1980s, conceptions of models originally rooted in cognitive science have been supplemented with philosophical arguments (Gilbert \& Justi 2016). Knuuttila (2011) argues that models are tools for generating knowledge. As tools, models consist of representations that are expressed externally via a material medium, e.g., ink and paper. Mental models, on the other hand, can be precursors to external representations, and are also part of the modeling process (Gilbert \& Justi 2016). To facilitate knowledge generation, models support people to ask questions, recognize and explain patterns in data, and make judgements about those explanations (Passmore \& Svoboda 2012). Models can also be used to communicate new knowledge or understanding to others (Schwarz 2009). However, tools do not spontaneously work on their own. Indeed, Giere (2009) and Gouvea \& Passmore (2017) make bids for focusing on the agents and ends of modeling: people use models to represent part of the world for some purpose.

In physics education, the purpose of modeling is often for students to describe, explain, or predict the behavior of physical phenomena (Etkina et al 2006). Students use evidence to generate models, and they use models to search for new evidence. Such intertwining of evidence- and model-based reasoning supports students to make sense of phenomena (Russ \& Odden 2017). Koponen (2007) argues that modeling in physics is a bidirectional process through which models both inform, and are informed by, experimental apparatus. Apparatus are designed to isolate particular aspects of a model, and data are used to verify the model or identify its limitations. Koponen further claims that two models are at play during experimentation: one to formulate explanations or predictions about a physical system that is the target of investigation, and another to interpret the experimental data generated by the measurement equipment. Thus, in physics education, the practice of modeling should involve recursive interactions between apparatus, evidence, and models of both phenomena and equipment. More broadly, we view such interactions as key features of experimental physics practice. Although many theoretical frameworks for modeling exist (Brewe 2008, Fuhrmann et al 2018, Gilbert \& Justi 2016, Passmore \& Svoboda 2012, Schwarz 2009, Windschitl et al 2008), few capture these key features.

Here, we discuss the Modeling Framework for Experimental Physics, which was explicitly designed to characterize physicists' use of models when conducting experiments. In the next section, we describe the Framework using a Malus's Law experiment to explicate various modeling subtasks and processes. Then, in Secs. 3 and 4. we provide an overview of the Framework's initial development and its subsequent 
validation and use as a research tool. Sec. 5 discusses applications to the design of undergraduate lab courses. Finally, in Sec. 6, we argue that the Modeling Framework is a dual-purpose tool that is theoretically sound, well-tested, and versatile. By "versatile," we mean that the Framework is applicable to multiple physics domains, it can be used to characterize student and instructor behaviors, and it can inform the design and evaluation of curricula for lab courses.

\section{Description of the Modeling Framework for Experimental Physics}

A visual representation of the Modeling Framework for Experimental Physics is given in Fig. 1. the Framework can be thought of as a flowchart that consists of five subtasks: making measurements, constructing models of equipment and phenomena, making comparisons between data and predictions, proposing causes for discrepancies, and enacting revisions to models and apparatus. Because modeling is a recursive process, the flowchart is cyclical. In the Framework, the goal is to achieve sufficiently good agreement between data and predictions, consistent with other work on modeling in physics education (Etkina et al 2006).

Below, we elaborate on each subtask of the Modeling Framework, drawing on examples from a Malus's Law experiment to explicate abstract concepts. This experiment involves shining laser light through two linear polarizers, one that is fixed and one that is free to rotate (the analyzer). After passing through both polarizers, the intensity of the transmitted laser light is proportional to $\cos ^{2} \theta$, where $\theta$ is the angle between the axes of polarization of the two polarizers. Thus, by rotating the analyzer, students can vary the intensity of the transmitted light. A schematic of the experiment is provided in Fig. 2 ,

(i) Make measurements. The measurement equipment interacts with the physical system and produces raw data.

Malus's Law example. The physical system apparatus includes a laser and two linear polarizing filters. The measurement equipment consists of a photodetector and voltmeter. Making measurements involves shining light through the polarizers onto the photodetector and producing a voltage on the voltmeter. See Fig. 2(A).

(ii) Construct models. The physical and measurement system apparatus are associated with distinct models. Each model is created from the key features of the system, relevant physics principles and concepts, particular parameter values, and appropriate assumptions and simplifications that make the model tractable, while limiting its scope of applicability.

Malus's Law example. The simplest model of the physical system involves treating the laser light as a perfectly monochromatic plane wave with uniform intensity. Polarizers are similarly idealized as having extinction ratios of exactly zero. The simplest measurement system model treats the measurement equipment as a black box: the photodetector instantaneously converts incident laser light to an electrical 


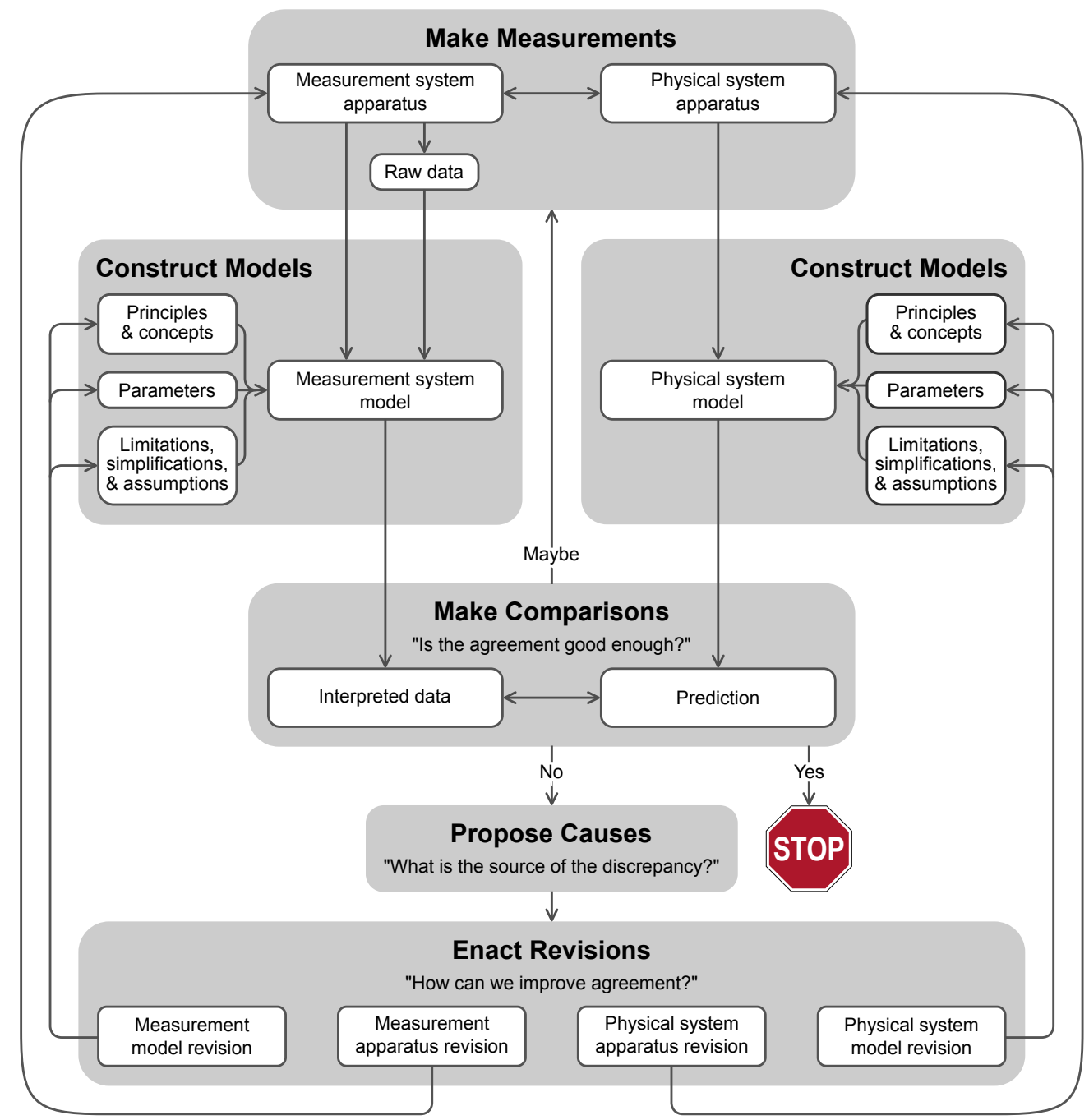

Figure 1. Modeling Framework for Experimental Physics. The version of the Framework presented here was previously published by Dounas-Frazer et al (2018) and is adapted from a visualization originally developed by Zwickl et al (2014).

signal that is sent to the voltmeter, which produces a voltage linearly proportional to the power of the light.

(iii) Make comparisons. The physical system model is used to generate predictions, and the measurement system model is used to analyze and interpret raw data. Interpreted data are compared to predictions, and domain-specific criteria are used to determine whether the level of agreement is good enough. Applying accepted scientific criteria for determining what counts as "good enough" is part of the modeling process (Giere 2009, Gouvea \& Passmore 2017). If the agreement is good enough, then there is no need to revise models or apparatus. However, additional data are sometimes needed in order to properly judge the quality of agreement; in this case, more measurements are required. If there is a discrepancy between data and predictions, the experimenter may propose and enact changes to models or apparatus to resolve the discrepancy. In this sense, the "Maybe" and 

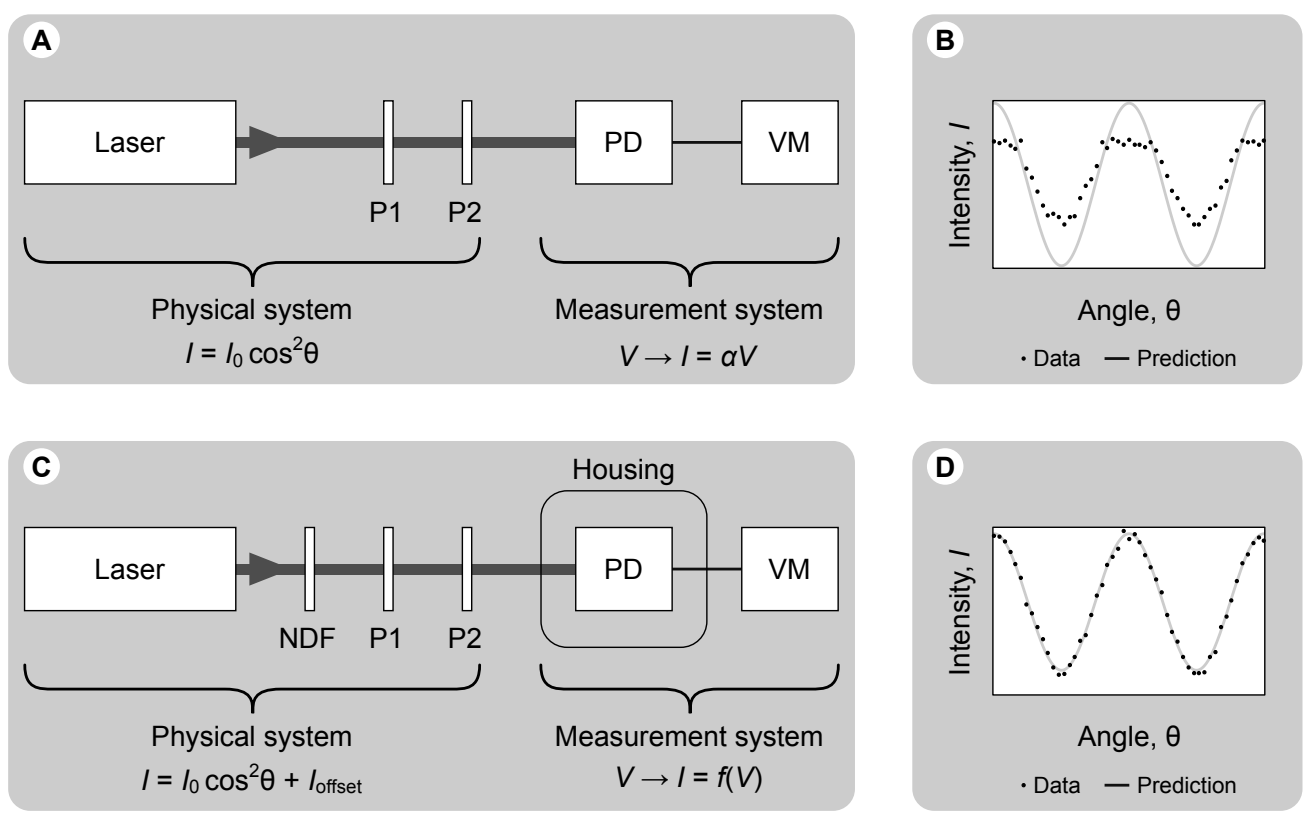

Figure 2. Hypothetical Malus's Law experiment. (A) Initial setup. P1 and P2 are polarizers. PD is a photodetector. VM is a voltmeter. $I_{0}$ and $I$ are the intensities of laser light incident on P2 and PD. $\theta$ is the angle between axes of polarization of P1 and P2. $\alpha$ is related to the linear responsivity of the PD. (B) Disagreement between data and prediction. (C) Revised setup. NDF is a neutral density filter. $I_{\text {offset }}$ is a modification to Malus's Law. $f(V)$ represents nonlinear calibration of PD output to optical power. (D) Improved agreement between data and prediction.

"No" pathways in Fig. 1 respectively correspond to efforts to reduce statistical and systematic sources of uncertainty.

Malus's Law example. The physical system model is used to predict Malus's Law: $I=I_{0} \cos ^{2} \theta$, where $I_{0}$ and $I$ are the intensities of light incident on the analyzer and photodetector, respectively. The measurement system model is used to interpret raw data by converting voltage as a function of angle to normalized intensity as a function of angle. Experimental data are compared to a cosine-squared curve; see Fig. 2(B). Goodness-of-fit metrics can help determine quality of agreement. Many such metrics have established criteria for acceptable fits among physicists.

(iv) Propose causes. When discrepancies between data and predictions must be resolved, physicists generate hypotheses about potential sources of those discrepancies. This modeling subtask can be challenging for people who are unfamiliar with nonideal behavior of equipment or assumptions that limit the predictive power of models (Zwickl et al 2015, Dounas-Frazer et al 2018). Malus's Law example. Common sources of discrepancy between data and prediction include nonzero background due to ambient light, polarizers with nonzero extinction ratios, or laser light with nonzero ellipticity. Discrepancies may also arise if the light is sufficiently powerful to saturate the photodetector or if changes in light power are faster than the slew rate limit of the detector. 
(v) Enact revisions. The Modeling Framework describes four pathways for revision: one may revise the models or apparatus of the physical or measurement systems. These pathways are consistent with other conceptions of modeling as a process that informs the design and execution of experiments (Gilbert \& Justi 2016, Koponen 2007, Russ \& Odden 2017). Depending on which proposed causes are deemed most likely or easiest to implement, some revisions may be prioritized over others (Stanley et al 2017). Once revisions are enacted, the modeling cycle repeats until data and predictions are brought into good enough agreement.

Malus's Law example. Apparatus revisions include covering the photodetector to shield it from ambient light, or placing a neutral density filter in the optical path of the laser light to decrease its overall intensity. Model revisions include adding a fit variable to Malus's Law to represent offsets due to ambient light or ellipticity, or determining the nonlinear calibration of the photodetector for high light intensities. See parts (C) and (D) of Fig. 2.

\section{Development of the Modeling Framework}

The Modeling Framework (Fig. 1) was originally developed in the context of the transformation of the Advanced Laboratory course at the University of Colorado Boulder (CU) (Zwickl et al 2013, Zwickl et al 2014). Those efforts began in late 2010 and employed the following approach: define learning goals, develop curriculum that align with those goals, and assess student learning. The Modeling Framework emerged from the first two phases of the transformation process. To identify consensus learning goals for the course, Zwickl et al (2013) worked with 21 faculty members via individual interviews or group meetings. Learning goals were also informed by comparison of the Advanced Laboratory to other laboratory courses and a review of the physics education literature. Using this process, Zwickl et al identified modeling as a major learning goal for the course. They further argued that modeling had three components: modeling the physical system, modeling the measurement system, and comparing data and predictions.

After identifying modeling as a learning goal for the Advanced Laboratory, Zwickl et al (2014) developed laboratory activities designed to engage students in the practice of modeling during optics experiments (Sec. 5). To guide their designs, they created the first iteration of the Modeling Framework. This iteration was informed by a review of the physics education literature and Zwickl and Lewandowski's own experience and expertise as experimental physicists. The first iteration differs from that presented in Fig. 1 in several ways, most of them aesthetic. Nevertheless, the main features have persisted across all versions of the Framework: measurement and physical systems are distinct, revisions include changes to both apparatus and models, and the need for revision is triggered by comparisons that fail to yield "good enough" agreement between interpreted data and model predictions. Thus, from its inception, the Modeling Framework aligned with philosophical conceptions of modeling, such as Koponen's (2007) bidirectionality. 


\section{Validation and use of the Modeling Framework as a research tool}

After the Modeling Framework was developed, it was validated and used as a research tool in a series of four studies, three focused on students (Dounas-Frazer et al 2016, Stanley et al 2017, Zwickl et al 2015) and one on instructors (Dounas-Frazer et al 2018). These studies demonstrate that the Modeling Framework is appropriate for characterizing modeling in upper-division lab contexts. They also resulted in small changes that improved the descriptive power of the Framework.

\subsection{Student approaches to modeling during optics and electronics activities}

Zwickl et al (2015) conducted think-aloud interviews during which eight students verbalized their reasoning while testing the power output of a light-emitting diode. One major contribution of this investigation was validation of the Modeling Framework's focus on measurement system models. During interviews, student engagement with the measurement apparatus (i.e., a photodetector and oscilloscope) included constructing models, revising models, interpreting output, and identifying limitations of the equipment. Zwickl et al also described two common barriers to students' use or revision of models. First, some students did not articulate crucial assumptions and corresponding limitations of their models, preventing them from making model-based refinements to their experiment. Second, lack of familiarity with the concept of solid angle and the unit of steradian prevented some students from appropriately comparing their measurements to numerical values specified in the data sheet for the light-emitting diode. These barriers are consistent with other work that has demonstrated how students' prior knowledge impacts their ability to construct and evaluate models (Fortus et al 2016, Ruppert et al 2017, Stewart et al 2005).

Next, Dounas-Frazer et al (2016) expanded on previous work by demonstrating that the Modeling Framework is applicable to a context other than optics, namely, electronics. In that work, eight pairs of students from two institutions participated in think-aloud interviews that involved troubleshooting a malfunctioning electric circuit. Troubleshooting can be thought of as a type of modeling in which the physical circuit is revised in order to bring its performance into alignment with expectations informed by a model. Dounas-Frazer et al examined students' modeling behaviors during two key episodes in the troubleshooting process: isolating the source of malfunction to a particular subsystem, and evaluating the performance of the repaired circuit. In the former episode, students engaged in constructing models and making comparisons

more often than other subtasks; in the latter episode, making comparisons, proposing causes, and enacting revisions were the most common subtasks. Thus, not only did students engage in multiple modeling cycles throughout the troubleshooting process, different phases of troubleshooting corresponded to different combinations of subtasks. Because troubleshooting is a central feature of electronics lab courses (Dounas-Frazer \& Lewandowski 2017), this study suggests that so, too, is modeling.

Transitioning from clinical research settings to an actual classroom, Stanley et 
al (2017) used the Modeling Framework to study student use of models in an Electronics Laboratory course specifically designed to engage students in modeling circuits (Lewandowski \& Finkelstein 2015). They analyzed 45 student lab notebooks for evidence of students' documented engagement in modeling subtasks. Three notebook entries were selected for analysis, each corresponding to a different activity: voltage divider circuit (high scaffolding), photometer circuit (medium scaffolding), and voltagecontrolled electromagnet (low scaffolding). Student engagement in modeling tracked the level of scaffolding in the lab guide. Compared to open-ended prompts, explicit prompts resulted in more thorough engagement in modeling. In particular, most students did not enact revisions to their models or apparatus unless specifically asked to do so. Further, Stanley et al found that students often neglected to make and document comparisons between expected and actual performance of circuits even when prompted, potentially due to lack of clarity about what constitutes "good enough" agreement between data and predictions. Making comparisons and judging the quality of agreement is crucial for making informed decisions about whether and how to iteratively improve an experiment (Holmes et al 2015). Therefore, there is a clear need to better understand and support student reasoning about which standards for "good enough" agreement are appropriate in different experimental physics contexts (cf. Giere 2009, Gouvea \& Passmore 2017). In addition to identifying areas for curricular improvement and future study, Stanley et al demonstrated that the Modeling Framework is useful for understanding student modeling in formal educational settings.

\subsection{Instructor perspectives on modeling in optics and electronics lab courses}

Shortly after it was created, the Modeling Framework informed a set of nationally endorsed recommendations for undergraduate physics lab courses in the United States (AAPT Committee on Laboratories 2015). However, until recently, research on modeling in upper-division physics labs focused almost exclusively on the perspectives and behaviors of instructors and students from CU (Dounas-Frazer et al 2016, Stanley et al 2017, Zwickl et al 2014, Zwickl et al 2015).

To get a better idea of whether and how modeling is taken up in lab courses across the United States, Dounas-Frazer et al (2018) conducted interviews with 19 optics instructors and 16 electronics instructors from 27 institutions. During these interviews, instructors described how various subtasks of the Modeling Framework aligned with their learning goals or activity design. Making measurements, constructing models, and comparing data to predictions were each identified as important learning goals by a majority of instructors in the study. Enacting revisions and proposing causes were less commonly identified as important. Limited class time was cited as a barrier to student revisions to experiments. Meanwhile, many instructors said that students are unfamiliar with the nonideal behavior of devices, and therefore struggle to propose causes for discrepancies between data and predictions. This finding is consistent with students' failure to recognize or articulate assumptions and limitations of models during 
experimental physics think-aloud interviews (Zwickl et al 2015).

Further, Dounas-Frazer et al (2018) found that modeling is taken up differently in optics compared to electronics. For example, with respect to the subtask of making comparisons, optics instructors were more likely to describe engaging students in rigorous statistical analyses (e.g., fitting curves to data and evaluating the goodness of fit). In contrast, electronics instructors said that comparisons typically involved qualitative checks of circuit performance because building functional circuits was more important than achieving precise agreement between predicted and expected output voltages. Electronics instructors often framed particular modeling subtasks or the whole Modeling Framework as necessary aspects of troubleshooting, in alignment with students' approaches to repairing malfunctioning circuits during think-aloud interviews (Dounas-Frazer et al 2016). Overall, Dounas-Frazer et al (2018) not only demonstrated the versatility of the Framework for describing the goals and activities of a national

sample of lab courses, they also shed light on the different purposes of modeling across two experimental physics domains.

\subsection{Empirically motivated changes to the Modeling Framework}

Over the course of the investigations described in Secs. 4.1 and 4.2, the Modeling Framework itself was modified in order to better capture the experimental modeling process. While many changes were aesthetic, some represented shifts in understanding of what modeling entails. In the first visualization of the Framework, Zwickl et al (2014) did not explicitly include "limitations" as part of system models, even though they recognized that students should be able to articulate model limitations. After Zwickl et al (2015) observed that students' unarticulated assumptions and unrecognized model limitations were barriers to modeling, they revised the Framework to include "limitations, simplifications, and assumptions" as an explicit part of system models. The "Maybe" pathway was added by Dounas-Frazer et al (2018) based on experience observing students troubleshoot electric circuits (Dounas-Frazer et al 2016). When confronted with a disagreement between data and predictions, students sometimes collected additional data in order to be sure that the discrepancy was significant, rather than immediately trying to explain it. These and other empirically motivated changes to the Modeling Framework likely improve its usefulness as a well-tested tool for describing the experimental modeling process.

\section{Applications of the Modeling Framework to curriculum design}

The Modeling Framework (Fig. 1) is a dual-purpose tool that can both characterize people's reasoning about experimental systems and inform curriculum design for laboratory courses (Zwickl et al 2014). To date, we are aware of applications of the Framework to the design of three undergraduate contexts: a fourth-year Advanced Laboratory (Zwickl et al 2014), a third-year Electronics Laboratory (Lewandowski \& 
Finkelstein 2015), and first-year introductory courses (Vonk et al 2017).

The first educational application of the Modeling Framework coincided with its development, as described in Sec. 3. After establishing that students' ability to model experimental systems was a learning goal for the CU Advanced Laboratory (Zwickl et al 2013), Zwickl et al (2014) developed and used the Framework to guide their transformation of that course. Soon after, Lewandowski \& Finkelstein (2015) also transformed the $\mathrm{CU}$ Electronics Laboratory in order to meet similar learning goals. Both courses aimed to engage students in constructing models, making predictions and comparisons, and revising models and apparatus. Inspired by previous work on scaffolded inquiry (Buck et al 2008, Etkina et al 2008, Hmelo-Silver et al 2007, Kirschner et al 2006), lab guides explicitly prompted students to engage in these modeling subtasks. For example, the lab guide for an Advanced Laboratory polarization activity prompted students to use the Jones formalism to model the propagation of laser light through polarizing filters and wave plates, and to derive predictions like Malus's Law (Zwickl et al 2014). To model the measurement system, students used manufacturer documentation to understand the operation and limitations of a photodetector, and to appropriately convert output voltage into measurements of optical power. Similarly, in the Electronics Laboratory, the lab guide for a voltage divider circuit prompted students to revise their circuit schematic and equation for the transfer function in order to include the input resistance of a digital multimeter. The level of explicit scaffolding in the lab guides faded over the course of the semester in order to provide students with more control over how they modeled their circuits (Lewandowski \& Finkelstein 2015). In both courses, lab guides prompted students to reason about model limitations or revisions in order to explain or minimize systematic biases in their measurements.

At the introductory level, Vonk et al (2017) used the Modeling Framework to design model-making activities in introductory algebra- and calculus-based physics courses. Here "model-making" refers to devising an experiment to determine the relationship between two variables (e.g., wavelength and frequency of a wave on a string), collecting and analyzing data, constructing a model to relate the variables, and using the model to make predictions. Instead of working with apparatus, students used Direct Measurement Videos (DMVs) to explore phenomena and collect data. DMVs are "short high-quality videos that show a scientifically interesting event," which are analyzed using online tools like digital rulers and stopwatches (Vonk et al 2017, p.4). Because students did not use apparatus, they engaged in only some modeling subtasks: making measurements, constructing models of physical phenomena, and making comparisons. In a study of 116 students' performance on a model-making assessment, Vonk et al showed that students who completed activities using DMVs designed to bolster model-making skills outperformed those who did not. This work suggests that the Modeling Framework has implications for a wide range of course formats and activity types beyond physics labs and apparatus-based activities. 


\section{Summary and ongoing work}

The Modeling Framework for Experimental Physics (Fig. 1) was developed to describe the process of constructing, using, and revising models when conducting physics experiments. It was informed by, and is consistent with, other theoretical conceptions of scientific modeling. Multiple studies have demonstrated that there is a good empirical mapping between the Framework and students' approaches to completing experimental physics tasks and instructors' learning goals and activity design in upper-division lab courses. Additionally, the Framework has been used to inform the design and evaluation of lab courses and introductory algebra- and calculus-based physics courses. For these reasons, we argue that the Modeling Framework is a theoretically sound, well-tested, and versatile tool.

The multi-year process through which the Modeling Framework was developed has yielded some insights about barriers to engaging students in modeling during upper-division physics labs. In these courses, students' prior knowledge about relevant physics concepts or model limitations impacts their ability to appropriately compare data to predictions or propose causes for discrepancies, and their ability to judge the quality of agreement between data and predictions impacts whether they revise their models or apparatus. We believe that it is possible to strike an effective balance between learning physics concepts and theories, and engaging in authentic modelingbased experimentation. However, the dearth of research on teaching and learning in physics labs (National Research Council 2012) makes it difficult to know which instructional practices work well for particular learning goals, physics domains, and student populations.

In ongoing work, we are using the Modeling Framework to inform the design of standardized and scalable assessments of students' experimental modeling abilities (Dounas-Frazer et al 2018). Interviews with instructors point to the need for processbased instruments that assess students' competence with multiple subtasks and the iterative nature of modeling, as well as their rationale for choosing one modeling pathway over another (e.g., deciding to revise the apparatus rather than collect more data). We aim to develop instruments that are compatible with a recent model for centralized data collection and large-scale deployment of research-based assessments (Wilcox et al 2016). Doing so will enable us to identify individual courses or types of courses that are successfully improving students' experimental modeling abilities, which in turn will pave the way for further research on effective modeling-oriented teaching practices in physics labs. Ultimately, we hope that this work will create more opportunities for students to authentically engage in experimentation during their undergraduate physics education.

\section{Acknowledgments}

We thank Benjamin Pollard and Laura Ríos for useful discussions about the ideas presented here. This material is based upon work supported by the National Science 
Foundation under Grant Nos. DUE-1726045 and PHY-1734006.

\section{References}

AAPT Committee on Laboratories 2015 AAPT Recommendations for the Undergraduate Physics Laboratory Curriculum American Association of Physics Teachers.

Brewe E 2008 'Modeling theory applied: Modeling Instruction in introductory physics' American Journal of Physics 76(12), 1155-1160.

Brewe E, Bartley J E, Riedel M C, Sawtelle V, Salo T, Boeving E R, Bravo E I, Odean R, Nazareth A, Bottenhorn K L, Laird R W, Sutherland M T, Pruden S M \& Laird A R 2018 'Toward a neurobiological basis for understanding learning in university Modeling Instruction physics courses' Frontiers in ICT $\mathbf{5}, 10$.

Buck L B, Bretz S L \& Towns M H 2008 'Characterizing the level of inquiry in the undergraduate laboratory' Journal of College Science Teaching 38(1), 52-58.

Coppens P, Van den Bossche J \& De Cock M 2016 'Goals of lab work in electronics: Student and staff ideas' International Journal of Electrical Engineering Education 53(2), 124-136.

Deacon C \& Hajek A 2011 'Student perceptions of the value of physics laboratories' International Journal of Science Education 33(7), 943-977.

Dounas-Frazer D R \& Lewandowski H J 2017 'Electronics lab instructors' approaches to troubleshooting instruction' Phys. Rev. Phys. Educ. Res. 13, 010102.

Dounas-Frazer D R, Ríos L, Pollard B, Stanley J T \& Lewandowski H J 2018 'Aligning assessment design with lab instructors' self-reported learning goals about experimental modeling skills' arXiv 1712, 1712.

Dounas-Frazer D R, Van De Bogart K L, Stetzer M R \& Lewandowski H J 2016 'Investigating the role of model-based reasoning while troubleshooting an electric circuit' Phys. Rev. Phys. Educ. Res. 12, 010137.

Etkina E, Karelina A \& Ruibal-Villasenor M 2008 'How long does it take? A study of student acquisition of scientific abilities' Phys. Rev. ST Phys. Educ. Res. 4, 020108.

Etkina E, Warren A \& Gentile M 2006 'The role of models in physics instruction' The Physics Teacher 44(1), 34-39.

Fortus D, Shwartz Y \& Rosenfeld S 2016 'High school students' meta-modeling knowledge' Research in Science Education 46(6), 787-810.

Fredlund T, Airey J \& Linder C 2012 'Exploring the role of physics representations: an illustrative example from students sharing knowledge about refraction' European Journal of Physics 33(3), 657 .

Fuhrmann T, Schneider B \& Blikstein P 2018 'Should students design or interact with models? Using the Bifocal Modelling Framework to investigate model construction in high school science' International Journal of Science Education $\mathbf{0}(0), 1-27$.

Giere R N 2009 'An agent-based conception of models and scientific representation' Synthese $\mathbf{1 7 2}(2), 269$.

Gilbert J K \& Justi R 2016 'Models of modelling' in 'Modelling-based Teaching in Science Education' Springer International Publishing Cham pp. 17-40.

Gouvea J \& Passmore C 2017 "Models of' versus 'Models for" Science 83 Education 26(1), 49-63.

Halloun I A \& Hestenes D 1987 'Modeling instruction in mechanics' American Journal of Physics $\mathbf{5 5}(5), 455-462$.

Hanif M, Sneddon P H, Al-Ahmadi F M \& Reid N 2009 'The perceptions, views and opinions of university students about physics learning during undergraduate laboratory work' European Journal of Physics 30(1), 85.

Hestenes D 1987 'Toward a modeling theory of physics instruction' American Journal of Physics $\mathbf{5 5}(5), 440-454$.

Hmelo-Silver C E, Duncan R G \& Chinn C A 2007 'Scaffolding and achievement in problem-based 
and inquiry learning: A response to Kirschner, Sweller, and and Clark (2006)' Educational Psychologist 42(2), 99-107.

Holmes N G, Wieman C E \& Bonn D A 2015 'Teaching critical thinking' Proceedings of the National Academy of Sciences 112(36), 11199-11204.

Johnson-Laird P 1980 'Mental models in cognitive science' Cognitive Science 4(1), 71-115.

Kirschner P A, Sweller J \& Clark R E 2006 'Why minimal guidance during instruction does not work: An analysis of the failure of constructivist, discovery, problem-based, experiential, and inquirybased teaching' Educational Psychologist 41(2), 75-86.

Knuuttila T 2011 'Modelling and representing: An artefactual approach to model-based representation' Studies in History and Philosophy of Science Part A 42(2), 262-271.

Koponen I T 2007 'Models and modelling in physics education: A critical re-analysis of philosophical underpinnings and suggestions for revisions' Science $\&$ Education 16(7-8), 751-773.

Körhasan N D, Eryılmaz A \& Şakir Erkoç 2016 'The influence of instructional interactions on students' mental models about the quantization of physical observables: a modern physics course case' European Journal of Physics 37(1), 015701.

Lewandowski H J \& Finkelstein N 2015 'Redesigning a junior-level electronics course to support engagement in scientific practices' in 'Physics Education Research Conference 2015' PER Conference College Park, MD pp. 191-194.

McPadden D \& Brewe E 2017 'Impact of the second semester university modeling instruction course on students' representation choices' Phys. Rev. Phys. Educ. Res. 13, 020129.

National Research Council 2012 Discipline-Based Education Research: Understanding and Improving Learning in Undergraduate Science and Engineering The National Academies Press Washington, DC.

Opfermann M, Schmeck A \& Fischer H E 2017 'Multiple representations in physics and science education - why should we use them?' in D. F Treagust, R Duit \& H. E Fischer, eds, 'Multiple Representations in Physics Education' Springer International Publishing Cham pp. 1-22.

Özcan O 2015 'Investigating students' mental models about the nature of light in different contexts' European Journal of Physics 36(6), 065042.

Passmore C M \& Svoboda J 2012 'Exploring opportunities for argumentation in modelling classrooms' International Journal of Science Education 34(10), 1535-1554.

Ruppert J, Duncan R G \& Chinn C A 2017 'Disentangling the role of domain-specific knowledge in student modeling' Research in Science Education pp. 1-28.

Russ R S \& Odden T O B 2017 'Intertwining evidence- and model-based reasoning in physics sensemaking: An example from electrostatics' Phys. Rev. Phys. Educ. Res. 13, 020105.

Schumacher D 2007 'Student undergraduate laboratory and project work' European Journal of Physics 28(3).

Schwarz C 2009 'Developing preservice elementary teachers' knowledge and practices through modelingcentered scientific inquiry' Science Education 93(4), 720-744.

Stanley J T, Su W \& Lewandowski H J 2017 'Using lab notebooks to examine students' engagement in modeling in an upper-division electronics lab course' Phys. Rev. Phys. Educ. Res. 13, 020127.

Stewart J, Cartier J L \& Passmore C M 2005 'Developing understanding through model-based inquiry' in S. M Donovan \& J. D Bransford, eds, 'How students learn' National Research Council Washington, DC pp. 515-565.

Vonk M, Bohacek P, Militello C \& Iverson E 2017 'Developing model-making and model-breaking skills using direct measurement video-based activities' Phys. Rev. Phys. Educ. Res. 13, 020106.

Wilcox B R \& Lewandowski H J 2018 'A summary of research-based assessment of students' beliefs about the nature of experimental physics' American Journal of Physics 86(3), 212-219.

Wilcox B R, Zwickl B M, Hobbs R D, Aiken J M, Welch N M \& Lewandowski H J 2016 'Alternative model for administration and analysis of research-based assessments' Phys. Rev. Phys. Educ. Res. 12, 010139.

Windschitl M, Thompson J \& Braaten M 2008 'Beyond the scientific method: Model-based inquiry as a 
new paradigm of preference for school science investigations' Science Education 92(5), 941-967.

Zwickl B M, Finkelstein N \& Lewandowski H J 2013 'The process of transforming an advanced lab course: Goals, curriculum, and assessments' American Journal of Physics 81(1), 63-70.

Zwickl B M, Finkelstein N \& Lewandowski H J 2014 'Incorporating learning goals about modeling into an upper-division physics laboratory experiment' American Journal of Physics 82(9), 876-882.

Zwickl B M, Hu D, Finkelstein N \& Lewandowski H J 2015 'Model-based reasoning in the physics laboratory: Framework and initial results' Phys. Rev. ST Phys. Educ. Res. 11, 020113. 